1 Hacettepe Journal of Mathematics and Statistics

$\bigcap$ Volume 45 (6) (2016), 1665-1673

\title{
On one problem of a cusped elastic prismatic shells in case of the third model of Vekua's hierarchical model
}

\author{
Natalia Chinchaladze*†
}

\begin{abstract}
In the present paper hierarchical model for cusped, in general, elastic prismatic shells is considered, when on the face surfaces a normal to the projection of the prismatic shell component of a traction vector and parallel to the projection of the prismatic shell components of a displacement vector are known.
\end{abstract}

Keywords: Cusped plates, cusped prismatic shells, degenerate elliptic systems, weighted spaces, Hardy's inequality, Korn's weighted inequality.

2000 AMS Classification: 74K20; 35J70

Received : 24.11.2015 Accepted : 02.03.2016 Doi : 10.15672/HJMS.20164516346

\section{Introduction}

Investigations of cusped elastic prismatic shells actually takes its origin from the fifties of the last century, namely, in 1955 I.Vekua raised the problem of investigation of elastic cusped prismatic shells, whose thickness on the prismatic shell entire boundary or on its part vanishes (see [15], [16], [9] and references therein).

Let $O x_{1} x_{2} x_{3}$ be an anticlockwise-oriented rectangular Cartesian frame of origin $O$. We conditionally assume the $x_{3}$-axis vertical. The elastic body is called a prismatic shell if it is bounded above and below by, respectively, the surfaces

$$
x_{3}=\stackrel{(+)}{h}\left(x_{1}, x_{2}\right) \text { and } x_{3}=\stackrel{(-)}{h}\left(x_{1}, x_{2}\right), \quad\left(x_{1}, x_{2}\right) \in \omega,
$$

laterally by a cylindrical surface $\Gamma$ of generatrix parallel to the $x_{3}$-axis and its vertical dimension is sufficiently small compared with other dimensions of the body. $\bar{\omega}:=\omega \cup \partial \omega$ is the so-called projection of the prismatic shell on $x_{3}=0$.

The main difference between the prismatic shell of a constant thickness and the standard shell of a constant thickness is the following: the lateral boundary of the standard

*I. Vekua Institute of Applied Mathematics \& Faculty of Exact and Natural Sciences of Iv. Javakhishvili Tbilisi State University, 2 University st., 0186 Tbilisi, Georgia, Email: chinchaladze@gmail.com

${ }^{\dagger}$ Corresponding Author. 
shell is orthogonal to the "middle surface" of the shell, while the lateral boundary of the prismatic shell is orthogonal to the prismatic shell's projection on $x_{3}=0$.

Let the thickness of the prismatic shell be

$$
2 h\left(x_{1}, x_{2}\right): \stackrel{\stackrel{(+)}{h}}{h}\left(x_{1}, x_{2}\right)-\stackrel{(-)}{h}\left(x_{1}, x_{2}\right) \begin{cases}>0 & \text { for }\left(x_{1}, x_{2}\right) \in \omega \\ \geq 0 & \text { for }\left(x_{1}, x_{2}\right) \in \partial \omega\end{cases}
$$

If the thickness of the prismatic shell vanishes on $\gamma_{0} \subset \partial \omega$, it is called cusped one.

Below we consider symmetric prismatic shell, i.e. the case when

$$
\stackrel{(+)}{h}\left(x_{1}, x_{2}\right)=-\stackrel{(-)}{h}\left(x_{1}, x_{2}\right),
$$

with the thickness as follows

$$
2 h:=h_{0} x_{2}^{\varkappa}, h_{0}, \varkappa=\text { const }, h_{0}, \varkappa>0 .
$$

I. Vekua [15], [16] constructed hierarchical models for elastic prismatic shells, in particular, plates of variable thickness, when on the face surfaces either es (the first model) or displacements (the second model) are known. The updated survey of results concerning cusped elastic prismatic shells in the cases of the first and second models is given in [9] (see also [1], [5], [6], [10], [12], [14] and references therein). In the present paper the third hierarchical model for cusped elastic prismatic shells is analyzed. It means that on the face surfaces a normal to the projection of the prismatic shell component $Q_{\left(\frac{ \pm}{\nu}\right)}$ of a traction vector and parallel to the projection of the prismatic shell components $u_{\alpha}\left(x_{1}, x_{2}, \stackrel{( \pm)}{h}, t\right)$ of a displacement vector are known. The third model was first suggested in [8].

In what follows the usual notations are used: $X_{i j}$ and $e_{i j}$ are the stress and strain tensors, respectively, $u_{i}$ are the displacements, $F_{i}$ are the volume force components, $\rho$ is the density, $\lambda$ and $\mu$ are the Lamé constants, $\delta_{i j}$ is the Kronecker delta, subscripts preceded by a comma mean partial derivatives with respect to the corresponding variables. Moreover, repeated indices imply summation (Greek letters run from 1 to 2 and Latin letters run from 1 to 3 ).

In the fifties of the twentieth century, I.Vekua ([9], [15], [16]) introduced a new mathematical model for elastic prismatic shells which was based on expansions of the three-dimensional displacement vector fields and the strain and stress tensors in linear elasticity into orthogonal Fourier-Legendre series with respect to the variable of plate thickness. By taking only the first $N+1$ terms of the expansions, he introduced the so-called $N$-th approximation. Each of these approximations for $N=0,1, \ldots$ can be considered as an independent mathematical model of plates. In particular, in case of the first model the approximations for $N=0$ and $N=1$ correspond to the plane deformation and classical Kirchhoff-Love plate model, respectively (see [9]).

For the sake of simplicity we consider zero approximation of the hierarchical model. Basic equation system can be written as follows (see e.g. [8], [3])

$$
\begin{aligned}
& \mu\left(h v_{\alpha 0}\right)_{, \beta \beta}+(\lambda+\mu)\left(h v_{\gamma 0}\right), \gamma \alpha \\
& -(\ln h)_{, \beta}\left\{\lambda \delta_{\alpha \beta}\left(h v_{\gamma 0}\right)_{, \gamma}+\mu\left[\left(h v_{\alpha 0}\right),_{\beta}+\left(h v_{\beta 0}\right)_{, \alpha}\right]\right\}+\Phi_{\alpha 0}=\rho h \ddot{v}_{\alpha 0}, \\
& \mu\left(h v_{30, \beta}\right)_{\beta}+\Phi_{30}=\rho h \ddot{v}_{30},
\end{aligned}
$$


where

$$
\begin{aligned}
& X_{\alpha \beta 0}\left(x_{1}, x_{2}, t\right)=\lambda \delta_{\alpha \beta}\left[\left(h v_{\gamma 0}\right)_{, \gamma}+\Psi_{\gamma \gamma}\right]+\mu\left[\left(h v_{\alpha 0}\right),_{\beta}+\left(h v_{\beta 0}\right)_{, \alpha}+2 \Psi_{\alpha \beta}\right], \\
& X_{3 \beta 0}\left(x_{1}, x_{2}, t\right)=\mu h v_{30, \beta}, \quad X_{330}=\lambda\left[\left(h v_{\gamma 0}\right)_{, \gamma}+\Psi_{\gamma \gamma}\right], \\
& e_{\alpha \beta 0}=\frac{1}{2}\left[\left(h v_{\alpha 0}\right)_{\beta}+\left(h v_{\beta 0}\right),_{\alpha}\right]+\Psi_{\alpha \beta}, \quad e_{3 \beta 0}=\frac{1}{2} h v_{30, \beta}, \quad e_{330}=0, \\
& \Phi_{\alpha 0}:=2 \mu \Psi_{\alpha \beta, \beta}+\lambda \Psi_{\gamma \gamma, \alpha}-(\ln h)_{, \beta}\left[\lambda \delta_{\alpha \beta} \Psi_{\gamma \gamma}+2 \mu \Psi_{\alpha \beta}\right]+F_{\alpha 0}, \\
& \Phi_{30}:=Q_{\left({ }_{\nu}\right)} \sqrt{\left(\stackrel{(+)}{h},{ }_{1}\right)^{2}+(\stackrel{(+)}{h}, 2)^{2}+1}+Q_{(-)} \sqrt{\left(\stackrel{(-)}{h},{ }_{1}\right)^{2}+(\stackrel{(-)}{h}, 2)^{2}+1}+F_{30} \text {, } \\
& \Psi_{\alpha \beta}:=\frac{1}{2}\left[u_{\beta}\left(x_{1}, x_{2}, \stackrel{(-)}{h}, t\right) \stackrel{(-)}{h}, \alpha-u_{\beta}\left(x_{1}, x_{2}, \stackrel{(+)}{h}, t\right) \stackrel{(+)}{h}, \alpha\right. \\
& \left.+u_{\alpha}\left(x_{1}, x_{2}, \stackrel{(-)}{h}, t\right) \stackrel{(-)}{h},{ }_{\beta}-u_{\alpha}\left(x_{1}, x_{2}, \stackrel{(+)}{h}, t\right) \stackrel{(+)}{h}, \beta\right],
\end{aligned}
$$

$X_{i j 0}, e_{i j 0}, u_{i 0}$ and $F_{i 0}$ are the zeroth order moments of $X_{i j}, e_{i j}, u_{i}$ and $F_{i}$, respectively; $v_{i 0}:=h^{-1} u_{i 0}$ are called weighted moments of the function $u_{i}$.

The case of cylindrical bending of the plates with the thickness (1.1) is considered in [8]. In this case the system (1.2)-(1.3) can be rewritten as follows

$$
\begin{gathered}
\mu\left(h\left(x_{2}\right) v_{10}\left(x_{2}\right)\right)_{, 22}-\mu\left(\ln h\left(x_{2}\right)\right)_{, 2}\left(h\left(x_{2}\right) v_{10}\left(x_{2}\right)\right)_{, 2}+\Phi_{10}\left(x_{2}\right)=0 \\
(\lambda+2 \mu)\left(h\left(x_{2}\right) v_{20}\left(x_{2}\right)\right),,_{22}-(\lambda+2 \mu)\left(\ln h\left(x_{2}\right)\right)_{, 2}\left(h\left(x_{2}\right) v_{20}\left(x_{2}\right)\right),_{2}+\Phi_{20}\left(x_{2}\right)=0, \\
\mu\left(h\left(x_{2}\right) v_{30,2}\left(x_{2}\right)\right),_{2}+\Phi_{30}\left(x_{2}\right)=0 .
\end{gathered}
$$

In [8] it is shown that $v_{\alpha 0}$ can not be prescribed in cusped edge (i.e., Dirichlet problem are not satisfied) if $\varkappa>0$, and $v_{30}$ can not be prescribed in cusped edge if $\varkappa \geq 1$.

The weak setting of the homogeneous Dirichlet problem of the following system

$$
\begin{aligned}
& \mu\left(h v_{\alpha 0}\right),_{\beta \beta}+(\lambda+\mu)\left(h v_{\gamma 0}\right)_{, \gamma \alpha} \\
& \quad-(\ln h)_{, \beta}\left\{\lambda \delta_{\alpha \beta}\left(h v_{\gamma 0}\right)_{, \gamma}+\mu\left[\left(h v_{\alpha 0}\right)_{, \beta}+\left(h v_{\beta 0}\right),_{\alpha}\right]\right\}+\Phi_{\alpha 0}=0, \\
& \mu\left(h v_{30, \beta}\right)_{\beta}+\Phi_{30}=0,
\end{aligned}
$$

is considered in [3].

\section{Vibration problem}

We will consider the case of harmonic vibration

$$
\begin{aligned}
v_{i 0}(x, t) & :=e^{-\iota \vartheta t} v_{i 0}^{0}(x), \quad \Phi_{i 0}(x, t):=e^{-\iota \vartheta t} \stackrel{0}{\Phi}_{i 0}(x), \quad \iota^{2}=-1, \\
\vartheta & =\text { const }>0, \quad x:=\left(x_{1}, x_{2}\right) \in \omega, \quad i=1,2,3 .
\end{aligned}
$$

Taking into account of (1.1), (1.2), and (1.3) for $v_{i 0}^{0}(x)$ we get the following system (the overscript index 0 is omitted below)

$$
\begin{aligned}
& -\rho \vartheta^{2} h v_{10}-\mu \Delta_{2}\left(h v_{10}\right)-(\lambda+\mu)\left[\left(h v_{10}\right),{ }_{11}+\left(h v_{20}\right), 21\right] \\
& +\mu(\ln h)_{, 2}\left[\left(h v_{10}\right)_{, 2}+\left(h v_{20}\right)_{1}\right]=\Phi_{10}, \\
& -\rho \vartheta^{2} h v_{20}-\mu \Delta_{2}\left(h v_{20}\right)-(\lambda+\mu)\left[\left(h v_{10}\right),{ }_{12}+\left(h v_{20}\right), 22\right] \\
& +(\ln h)_{, 2}\left\{\lambda\left[\left(h v_{10}\right)_{, 1}+\left(h v_{20}\right)_{, 2}\right]+2 \mu\left(h v_{20}\right)_{, 2}\right\}=\Phi_{20}, \\
& -\rho \vartheta^{2} h v_{30}-\mu\left[\left(h v_{30,1}\right)_{1}+\left(h v_{30,2}\right)_{2}\right]=\Phi_{30},
\end{aligned}
$$


where $\Delta_{2}$ is a two dimensional Laplace operator.

We can rewrite obtained system in the following vector form

$$
\mathbf{A} v(x)=\Phi(x), \quad x \in \omega,
$$

where

$$
\begin{gathered}
\mathbf{A}:=\left\|\begin{array}{ccc}
A_{11} & A_{12} & A_{13} \\
A_{21} & A_{22} & A_{23} \\
A_{31} & A_{32} & A_{33}
\end{array}\right\| \\
A_{11}:=-\rho \vartheta^{2} h-(\lambda+2 \mu) h \partial_{11}-\mu\left[h \partial_{22}+2 h,,_{2} \partial_{2}+h,,_{22}\right]+\mu(\ln h)_{,_{2}}\left[h \partial_{2}+h,,_{2}\right], \\
A_{12}:=-(\lambda+\mu)\left[h \partial_{12}+h,_{2} \partial_{1}\right]+\mu(\ln h){ }_{2} h \partial_{1}, \\
A_{21}:=-(\lambda+\mu)\left[h \partial_{12}+h,_{2} \partial_{1}\right]+\lambda(\ln h),_{2} h \partial_{1}, \\
A_{22}:=-\rho \vartheta^{2} h-\mu h \partial_{11}-(\lambda+2 \mu)\left[h \partial_{22}+2 h,,_{2} \partial_{2}+h,,_{22}\right]+(\lambda+2 \mu)(\ln h)_{,_{2}}\left[h \partial_{1}+h,,_{2}\right], \\
A_{13}=A_{23}=A_{31}=A_{32}=0, \quad A_{33}:=-\rho \vartheta^{2} h-\mu h\left(\partial_{11}+\partial_{22}\right)+\mu h,_{2} \partial_{2}, \\
v:=\left(v_{10}, v_{20}, v_{30}\right)^{\top}, \quad \Phi:=\left(\Phi_{10}, \Phi_{20}, \Phi_{30}\right),
\end{gathered}
$$

the symbol $(\cdot)^{\top}$ means transposition.

Let

$$
v, v^{*} \in C^{2}(\omega) \cap C^{1}(\bar{\omega}), \quad v^{*}:=\left(v_{10}^{*}, v_{20}^{*}, v_{30}^{*}\right)^{\top},
$$

where $v$ and $v^{*}$ are arbitrary vectors of the above class. We obtain the following Green's formula

$$
\int_{\omega} \mathbf{A} v \cdot v^{*} d \omega=\mathbf{J}\left(v, v^{*}\right)-\int_{\partial \omega} X_{n} v \cdot v^{*} d \partial \omega=\int_{\omega} \Phi \cdot v^{*} d \omega .
$$

Here $n:=\left(n_{1}, n_{2}\right)$ is the inward normal to $\partial \omega$ :

$$
X_{n}:=\left\{X_{n 10}, X_{n 20}, X_{n 30}\right\},
$$

with

$$
\begin{aligned}
& X_{n i 0}=X_{i j 0} n_{j} \\
& \mathbf{J}\left(v, v^{*}\right):=\int_{\omega}-h \rho \vartheta^{2} v_{i 0} v_{i 0}^{*} d \omega+\int_{\omega} \frac{\lambda}{h}\left[\left(h v_{10}\right),_{1}\left(h v_{10}^{*}\right)_{1}+\left(h v_{20}\right)_{, 2}\left(h v_{20}^{*}\right), 2\right. \\
& \left.+\left(h v_{10}\right)_{, 1}\left(h v_{20}^{*}\right)_{, 2}+\left(h v_{20}\right)_{, 2}\left(h v_{10}^{*}\right)_{, 1}\right] d \omega+\int_{\omega} \frac{\mu}{h}\left[2 f\left(h v_{10}\right)_{, 1}\left(h v_{10}^{*}\right)_{, 1}\right. \\
& +\left(h v_{10}\right)_{,_{2}}\left(h v_{10}^{*}\right), 2_{2}+\left(h v_{20}\right)_{, 1}\left(h v_{10}^{*}\right)_{,_{2}}+\left(h v_{20}\right)_{, 1}\left(h v_{20}^{*}\right)_{, 1}+\left(h v_{10}\right)_{, 2}\left(h v_{20}^{*}\right)_{, 1} \\
& \left.+2\left(h v_{20}\right),_{2}\left(h v_{20}^{*}\right)_{, 2}+h v_{30,1} h v_{30,1}^{*}+h v_{30,2} h v_{30,2}^{*}\right] d \omega \\
& =\int_{\omega}-h \rho \vartheta^{2} v_{i 0} v_{i 0}^{*} d \omega+\int_{\omega} \frac{\lambda}{h}\left(h v_{\alpha 0}\right),_{\alpha}\left(h v_{\beta 0}^{*}\right),_{\beta} d \omega \\
& +\int_{\omega} \frac{\mu}{h}\left\{\left[\left(h v_{\alpha 0}\right),_{\beta}+\left(h v_{\beta 0}\right),_{\alpha}\right]\left[\left(h v_{\alpha 0}^{*}\right)_{, \beta}+\left(h v_{\beta 0}^{*}\right),_{\alpha}\right]+\left(h v_{30, \alpha}\right)\left(h v_{30, \alpha}^{*}\right)\right\} d \omega \\
& =\int_{\omega} a\left[-h^{2} \rho \vartheta^{2} v_{i 0} v_{i 0}^{*}+\lambda e_{k k 0}^{1}(v) e_{i i 0}^{1}\left(v^{*}\right)+2 \mu e_{i j 0}^{1}(v) e_{i j 0}^{1}\left(v^{*}\right)\right] d \omega,
\end{aligned}
$$

where

$$
a:=\frac{1}{h},
$$




$$
e_{i j 0}^{1}(v):= \begin{cases}\frac{1}{2}\left[\left(h v_{i 0}\right),_{j}+\left(h v_{j 0}\right),{ }_{i}\right], & i, j=1,2, \\ \frac{1}{2} h v_{i 0}, j, & i=3, j=1,2, \\ 0, & i=j=3 .\end{cases}
$$

If we consider BVPs for system (2.1) with homogeneous boundary conditions for which the curvilinear integralalong $\partial \omega$ in (2.2) disappears, we arrive at the equation

$$
\mathbf{J}\left(v, v^{*}\right)=\int_{\omega} \Phi \cdot v^{*} d \omega .
$$

Denote by $\mathcal{D}(\omega)$ a space of infinity differentiable functions with compact support in $\omega$ and introduce the linear form $[\mathcal{D}(\omega)]^{3}$ by the formula:

$$
\begin{aligned}
& \left(v, v^{*}\right)_{X_{\vartheta}^{\varkappa}}=\int_{\omega}\left[h^{2} \rho \vartheta^{2} v_{i 0} v_{i 0}^{*}+e_{i j 0}^{1}(v) e_{i j 0}^{1}\left(v^{*}\right)\right] \frac{1}{h} d \omega \\
& \|v\|_{X_{\vartheta}^{\varkappa}}^{2}=\int_{\omega}\left[h \rho \vartheta^{2} v_{i 0} v_{i 0}+\frac{1}{4 h}\left(4\left[\left(h v_{10}\right),\right]_{1}\right]^{2}\right. \\
& \left.\left.\left.\quad+4\left[\left(h v_{20}\right)_{, 2}\right]^{2}+2\left(\left(h v_{10}\right)\right)_{2}+\left(h v_{20}\right)_{1}\right)^{2}+2\left(h v_{30,1}\right)^{2}+2\left(h v_{30,2}\right)^{2}\right)\right] d \omega .
\end{aligned}
$$

$X_{\vartheta}^{\varkappa}$ is a Hilbert space.

The classical and weak setting of the homogeneous Dirichlet problem can be formulated as follows:

2.1. Problem. Find a 3 -dimensional vector $v$ in $\omega$ satisfying the system of differential equations (2.1) in $\omega$ and the homogeneous Dirichlet boundary condition

$$
[v(x)]^{+}=0, \quad x \in \partial \omega .
$$

2.2. Problem. Find a vector $v \in X_{\vartheta}^{\varkappa}$ satisfying the equality

$$
\mathbf{J}\left(v, v^{*}\right)=\left\langle\Phi, v^{*}\right\rangle \text { for all } v^{*} \in X_{\vartheta}^{\varkappa},
$$

here, the vector $\Phi$ belongs to the adjoint space $\left[X_{\vartheta}^{\varkappa}\right]^{*}$, and $\langle\cdot, \cdot\rangle$ denotes duality brackets between the spaces $\left[X_{\vartheta}^{\varkappa}\right]^{*}$ and $X_{\vartheta}^{\varkappa}$.

Further, we construct the vectors in $\Omega:=\left\{\left(x ; x_{3}\right): x \in \omega,-h(x)<x_{3}<h(x)\right\}$ :

$$
\begin{aligned}
w_{i}\left(x, x_{3}\right) & =\frac{1}{2} v_{i 0}(x), \quad i=1,2,3, \\
w_{i}^{*}\left(x, x_{3}\right) & =\frac{1}{2} v_{i 0}^{*}(x), \quad i=1,2,3 .
\end{aligned}
$$

It can be shown that

$$
J\left(w, w^{*}\right):=\int_{\Omega}\left[-\rho \vartheta^{2} w_{i} w_{i}^{*}+\sigma_{i j}(w) e_{i j}\left(w^{*}\right)\right] d \Omega=\mathbf{J}\left(v, v^{*}\right),
$$

where $w\left(x, x_{3}\right):=\left(w_{1}, w_{2}, w_{3}\right)$ and $w^{*}\left(x, x_{3}\right):=\left(w_{1}^{*}, w_{2}^{*}, w_{3}^{*}\right)$ are vectors and $J\left(w, w^{*}\right)$ is the bilinear form corresponding to the three-dimensional potential energy for the displacement vector $w$.

In view of the homogeneous Dirichlet boundary condition (2.3), if $\varkappa>1$, the following Hardy inequality holds (see [13], p. 69; [11])

$$
\int_{\varepsilon}^{l} x_{2}^{\varkappa-2} v_{\alpha 0}^{2} d x_{2} \leq \frac{4}{(\varkappa-1)^{2}} \int_{\varepsilon}^{l} x_{2}^{\varkappa}\left(v_{\alpha 0,2}\right)^{2} d x_{2}, \quad \varkappa>1 .
$$


Replacing in $(2.6) \varkappa$ by $\varkappa+2$, we obtain

$$
\int_{\varepsilon}^{l} x_{2}^{\varkappa} v_{\alpha 0}^{2} d x_{2} \leq \frac{4}{(\varkappa+1)^{2}} \int_{\varepsilon}^{l} x_{2}^{\varkappa+2}\left(v_{\alpha 0,2}\right)^{2} d x_{2}, \text { for any } \varkappa>0 .
$$

Now, considering the limit procedure as $\varepsilon \rightarrow 0+$, since the limits of the integrals in (2.7) exist for $v_{\alpha 0} \in X_{\vartheta}^{\varkappa}$, we immediately get the following

$$
\int_{0}^{l} x_{2}^{\varkappa} v_{\alpha 0}^{2} d x_{2} \leq \frac{4}{(\varkappa+1)^{2}} \int_{0}^{l} x_{2}^{\varkappa+2}\left(v_{\alpha 0,2}\right)^{2} d x_{2} \text {, for any } \varkappa>0 .
$$

Integrating by $x_{1}$ both side of $(2.8)$ over $] x_{1}^{0}, x_{1}^{1}[$, we get

$$
\int_{\omega} x_{2}^{\varkappa} v_{\alpha 0}^{2} d \omega \leq \frac{4}{(\varkappa+1)^{2}} \int_{\omega} x_{2}^{\varkappa+2}\left(v_{\alpha 0,2}\right)^{2} d \omega, \text { for any } \varkappa>0
$$

2.3. Lemma. The bilinear form $\mathbf{J}(\cdot, \cdot)$ is bounded and strictly coercive in the space $X_{\vartheta}^{\varkappa}(\omega)$, i.e., there are positive constant $C_{0}$ and $C_{1}$ such that

$$
\left|\mathbf{J}\left(v, v^{*}\right)\right| \leq C_{1}\|v\|_{X_{\vartheta}^{\varkappa}}\left\|v^{*}\right\|_{X_{\vartheta}^{\varkappa}}
$$

$$
\mathbf{J}(v, v) \geq C_{0}\|v\|_{X_{\vartheta}^{\varkappa}}^{2}
$$

for all $v, v^{*} \in X_{\vartheta}^{\varkappa}$ and $\vartheta^{2}<\frac{\mu(\varkappa+1)^{2}}{16 \rho l^{2}}$.

Proof. In view of (2.5) we have

$$
\begin{aligned}
& \left|\mathbf{J}\left(v, v^{*}\right)\right|^{2}=\left|J\left(w, w^{*}\right)\right|^{2} \\
& =\left[\int_{\Omega}-\rho \vartheta^{2} w_{i} w_{i}^{*}+\left(2 \mu e_{i j}(w)+\lambda \delta_{i j} e_{k k}(w)\right) e_{i j}\left(w^{*}\right) d \Omega\right]^{2} \\
& \leq\left|\int_{\Omega} \rho \vartheta^{2} w_{i} w_{i}^{*} d \Omega\right|^{2}+C_{3}\left|\int_{\Omega}\left(2 \mu e_{i j}(w)+\lambda \delta_{i j} e_{k k}(w)\right) e_{i j}\left(w^{*}\right) d \Omega\right|^{2} \\
& \leq\left|\int_{\omega} h \rho \vartheta^{2} v_{i 0} v_{i 0}^{*} d \omega\right|^{2}+C_{2} \sum_{i, j=1}^{3} \int_{\Omega} e_{i j}^{2}(w) d \Omega \sum_{i, j=1}^{3} \int_{\Omega} e_{i j}^{2}\left(w^{*}\right) d \Omega \\
& \leq\left|\int_{\omega} h \rho \vartheta^{2} v_{i 0} v_{i 0}^{*} d \omega\right|^{2}+C_{2} \int_{\omega} \frac{1}{2} \sum_{i, j=1}^{3} e_{i j 0}^{2}(v) \frac{d \omega}{h} \int_{\omega} \frac{1}{2} \sum_{i, j=1}^{3} e_{i j 0}^{2}\left(v^{*}\right) \frac{d \omega}{h} \\
& \leq \int_{\omega} h \rho \vartheta^{2} \sum_{i=1}^{3} v_{i 0}^{2} d \omega \int_{\omega} h \rho \vartheta^{2} \sum_{i=1}^{3} v_{i 0}^{* 2} d \omega \\
& +C_{2} \int_{\omega} \frac{1}{2} \sum_{i, j=1}^{3} e_{i j 0}^{2}(v) \frac{d \omega}{h} \int_{\omega} \frac{1}{2} \sum_{i, j=1}^{3} e_{i j 0}^{2}\left(v^{*}\right) \frac{d \omega}{h} \\
& \leq C_{1}\|v\|_{X}^{2}\left\|v^{*}\right\|_{X \vartheta}^{2},
\end{aligned}
$$

where

$$
C_{1}:=\max \left\{1, C_{2}\right\}
$$

Whence (2.10) follows. 
Further, taking into account of (2.9) and of the fact that $2 \lambda+3 \mu>0, \mu>0$ we get

$$
\begin{aligned}
& \|v\|_{X_{\vartheta}}^{2} \leq \frac{\mathbf{J}(v, v)}{2 \mu}+\frac{\vartheta^{2} \rho h_{0}}{\mu} \int_{\omega} x_{2}^{\varkappa} v_{i 0}^{2} d \omega \leq \frac{\mathbf{J}(v, v)}{2 \mu}+\frac{4 \vartheta^{2} \rho h_{0}}{\mu(\varkappa+1)^{2}} \int_{\omega} x_{2}^{\varkappa+2}\left(v_{i 0,2}\right)^{2} d \omega \\
& \leq \frac{\mathbf{J}(v, v)}{2 \mu}+\frac{4 \vartheta^{2} \rho h_{0} l^{2}}{\mu(\varkappa+1)^{2}} \int_{\omega} x_{2}^{\varkappa}\left(v_{i 0,2}\right)^{2} d \omega \leq \frac{\mathbf{J}(v, v)}{2 \mu}+\frac{4 \vartheta^{2} \rho l^{2}}{\mu(\varkappa+1)^{2}} \int_{\omega} \frac{\left(h v_{i 0,2}\right)^{2}}{h} d \omega \\
& =\frac{\mathbf{J}(v, v)}{2 \mu}+\frac{4 \vartheta^{2} \rho l^{2}}{\mu(\varkappa+1)^{2}} \int_{\omega}\left[\frac{\left(h v_{10,2}\right)^{2}}{h}+\frac{\left(h v_{20,2}\right)^{2}}{h}+\frac{\left(h v_{30,2}\right)^{2}}{h}\right] d \omega \\
& \leq \frac{\mathbf{J}(v, v)}{2 \mu}+\frac{2 \vartheta^{2} \rho l^{2}}{\mu(\varkappa+1)^{2}} \int_{\omega}\left[\frac{2\left(h v_{10,2}\right)^{2}}{h}+\frac{4\left(h v_{20,2}\right)^{2}}{h}+\frac{2\left(h v_{30,2}\right)^{2}}{h}\right] d \omega \\
& \leq \frac{\mathbf{J}(v, v)}{2 \mu}+\frac{2 \vartheta^{2} \rho l^{2}}{\mu(\varkappa+1)^{2}} \int_{\omega}\left[\frac{2\left[\left(h v_{10}\right)_{2}\right]^{2}}{h}+\frac{4\left[\left(h v_{20}\right)_{2}\right]^{2}}{h}+\frac{2\left(h v_{30,2}\right)^{2}}{h}\right] d \omega \\
& \leq \frac{\mathbf{J}(v, v)}{2 \mu}+\frac{8 \vartheta^{2} \rho l^{2}}{\mu(\varkappa+1)^{2}}\|v\|_{X_{\vartheta}^{\varkappa},}^{2}
\end{aligned}
$$

from here we have

$$
\mathbf{J}(v, v) \geq\left(2 \mu-\frac{16 \vartheta^{2} \rho l^{2}}{(\varkappa+1)^{2}}\right)\|v\|_{X_{\vartheta}^{\varkappa}}^{2} .
$$

If we assume $\vartheta^{2}<\frac{\mu(\varkappa+1)^{2}}{16 \rho l^{2}}$ inequality (2.11) immediately follows from (2.12).

2.4. Remark. If $\mathbf{J}(v, v)=0$, then $v \equiv 0$ by (2.12).

2.5. Theorem. Let $F \in\left[X_{\vartheta}^{\varkappa}\right]^{*}$. Then the variational problem (2.4) has a unique solution $v \in X_{\vartheta}^{\varkappa}$ for an arbitrary value of the parameter $\varkappa$ and $\|v\|_{X_{\vartheta}} \leq \frac{1}{C_{0}}\|F\|_{\left[X_{\vartheta}\right]^{*}}$.

Proof. The proof can be realized by means of Lax-Milgram theorem (see Appendix A.1).

It can be easily shown that if $\Phi \in[L(\omega)]^{3}$ and $\operatorname{supp} \Phi \cap \bar{\gamma}_{0}=\emptyset$, then $\Phi \in\left[X_{\vartheta}^{\varkappa}\right]^{*}$ and

$$
\left\langle\Phi, v^{*}\right\rangle=\int_{\omega} \Phi(x) v^{*}(x) d \omega,
$$

since $v^{*} \in\left[H^{1}\left(\omega_{\varepsilon}\right)\right]^{3}$, where $\varepsilon$ is sufficiently small positive number such that supp $\Phi \subset$ $\omega_{\varepsilon}=\omega \cap\left\{x_{2}>\varepsilon\right\}$. Therefore,

$$
\begin{aligned}
& \left|\left\langle\Phi, v^{*}\right\rangle\right|=\left|\int_{\omega} \Phi(x) v^{*}(x) d \omega\right| \leq\|\Phi\|_{\left[L_{2}(\omega)\right]^{3}}\left\|v^{*}\right\|_{\left[L_{2}\left(\omega_{\varepsilon}\right)\right]^{3}} \\
& \leq\|\Phi\|_{\left[L_{2}(\omega)\right]^{3}}\left\|v^{*}\right\|_{\left[H^{1}\left(\omega_{\varepsilon}\right)\right]^{3}} \leq C_{\varepsilon}\|\Phi\|_{\left[L_{2}(\omega)\right]^{3}}\left\|v^{*}\right\|_{X_{\vartheta}^{\varkappa} .}
\end{aligned}
$$

In this case, we obtain the estimate

$$
\|v\|_{X_{\vartheta}} \leq \frac{C_{\varepsilon}}{C_{0}}\|\Phi\|_{\left[L_{2}(\omega)\right]^{3}} .
$$

For establishing a representation of the space $X_{0}^{\varkappa}$ as a weighted Sobolev space, we introduce the following space:

$$
Y_{0}^{\varkappa}:=\left[\stackrel{0}{\left.W_{2, \varkappa}^{1}(\omega)\right]^{2}}\right.
$$

$\stackrel{0}{\stackrel{0}{W}} \underset{W_{2}^{1}, \varkappa}{\text { where }}(\omega)$ is a completion $\mathcal{D}(\omega)$ by means of the norm

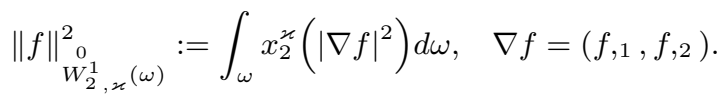


The norm in the space $Y_{0}^{\varkappa}$ for a vector $\left(v_{10}, v_{20}, v_{30}\right)$ reads as

$$
\|v\|_{Y_{0}^{\varkappa}}^{2}:=\int_{\omega} x_{2}^{\varkappa}\left(\sum_{\alpha=1}^{2}\left|\nabla v_{\alpha 0}\right|^{2}\right) d \omega .
$$

Using Korn's and Hardy's inequalities (see Appendix) the following theorem can be proved (similarly, to the Theorem 5.1 of [4])

2.6. Theorem. The linear spaces $X_{0}^{\varkappa}$ and $Y_{0}^{\varkappa}$ as sets of vector functions coincide and the norms $\|\cdot\|_{X_{0}^{\varkappa}},\|\cdot\|_{Y_{0}^{\varkappa}}$ are equivalent if $\varkappa=0$ and $\vartheta^{2}<\min \left\{\frac{\mu(\varkappa+1)^{2}}{16 \rho l^{2}}, \frac{2}{h_{0} \rho l^{2}}\right\}$.

2.7. Remark. Note that if $v \in X_{\vartheta}^{\varkappa}$, then all the components of $v$ posses the zero traces on part $\gamma_{1}$ of the boundary $\partial \omega$ for arbitrary $\varkappa$ due to the well-known trace theorem in the Sobolev space $W^{1}$. This follows, on the one hand, from the fact that the elliptic system under consideration is non-degenerated at the curve $\gamma_{1}$ and, on the other hand, from the construction of the space $X_{\vartheta}^{\varkappa}$.

\section{Appendix}

A.1. The Lax-Milgram theorem. Let $V$ be a real Hilbert space and let $J(w, v)$ be a bilinear form defined on $V \times V$. Let this form be continuous, i.e., let there exist a constant $K>0$ such that

$$
|J(w, v)| \leq K\|w\|_{V}\|v\|_{V}
$$

holds $\forall w, v \in V$ and $V$-elliptic, i.e., let there exist a constant $\alpha>0$ such that

$$
J(w, w) \geq \alpha\|w\|_{V}^{2}
$$

holds $\forall w \in V$. Further let $F$ be a bounded linear functional from $V^{*}$ dual of $V$. Then there exists one and only one element $z \in V$ such that

$$
J(z, v)=\langle F, v\rangle \equiv F v \quad \forall v \in V
$$

and

$$
\|z\|_{V} \leq \alpha^{-1}\|F\|_{V^{*}}
$$

Let $\omega$ be as in Section 1 and let $\mathcal{D}(\omega)$ be a space of infinitely differentiable functions with compact support in $\omega$.

A.2. Hardy's inequality. For every $f \in \mathcal{D}(\omega)$ and $\nu \neq 1$ there holds the inequality

$$
\int_{\omega} x_{2}^{\nu-2} f^{2}(x) d \omega \leq C_{\nu} \int_{\omega} x_{2}^{\nu}|\nabla f(x)|^{2} d \omega,
$$

where the positive constant $C_{\nu}$ is independent of $f$.

By completion of $\mathcal{D}(\omega)$ with the norm

$$
\|f\|_{W_{2, \nu}^{1}(\omega)}^{2}:=\int_{\omega} x_{2}^{\nu}|\nabla f(x)|^{2} d \omega,
$$

we conclude that the inequality (A.1) holds for arbitrary $f \in \stackrel{\circ}{W_{2, \nu}^{1}}(\omega)$.

For proof see [7].

A.3. Korn's weighted inequality. Let $\varphi=\left(\varphi_{1}, \varphi_{2}\right) \in\left[\stackrel{\circ}{{ }^{1}}{ }_{2, \nu}(\omega)\right]^{2}$ and $\nu \neq 1$. Then

$$
\int_{\omega} x_{2}^{\nu}\left[\left|\nabla \varphi_{1}(x)\right|^{2}+\left|\nabla \varphi_{2}(x)\right|^{2}\right] d \omega
$$




$$
\leq C_{\nu} \int_{\omega} x_{2}^{\nu}\left[\varphi_{1,1}^{2}(x)+\varphi_{2,2}^{2}(x)+\left(\varphi_{1,2}(x)+\varphi_{2,1}(x)\right)^{2}\right] d \omega,
$$

where the positive constant $C_{\nu}$ is independent of $\varphi$.

The proof can be found in [7], [17].

Acknowledgements. The present work was supported by the Shota Rustaveli National Science Foundation within the framework of the projects D-13/18 and 30/28.

\section{References}

[1] Babuška, I. and Li, L. Hierarchic modeling of plates, Comput. \& Structures 40, 419-430, 1991.

[2] Chinchaladze, N. Harmonic vibration of cusped plates in the $N$-th approximation of Vekua's hierarchical models, Archives of Mechanics 65 (5), 345-365, 2013.

[3] Chinchaladze, N. On some analytic methods for calculating of cusped prismatic shells, PAMM: Proceedings in Applied Mathematics and Mechanis 14 (1), 185-186, 2014.

[4] Chinchaladze, N., Jaiani, G., Gilbert, R., Kharibegashvili, R. and Natroshvili, D. Existence and uniqueness theorems for cusped prismatic shells in the $N$-th hierarchical model, Mathematical Methods in Applied Sciences 31 (11), 1345-1367, 2008.

[5] Chinchaladze, N., Jaiani, G., Gilbert, R., Kharibegashvili, R. and Natroshvili, D. Initialboundary value problems for solid-fluid composite structures, Zeitschrift Für Angewandte Mathematik und Physik (ZAMP) 63 (4), 625-653, 2012.

[6] Chinchaladze, N., Jaiani, G., Podio-Guidugli, P. and Maistrenko, B. Concentrated contact interactions in cuspidate prismatic shell-like bodies, Archive of Applied Mechanics 81 (10), 1487-1505, 2011.

[7] Devdariani, G., Jaiani, G., Kharibegashvili, R. and Natroshvili, D. The first boundary value problem for the system of cusped prismatic shells in the first approximation, Appl. Math. Inform. 5 (2), 26-46, 2000.

[8] Jaiani, G. Hierarchical models for prismatic shells with mixed conditions on face surfaces, Bulletin of TICMI 17 (2), 24-48, 2013.

[9] Jaiani, G. Cusped shell-like structures, SpringerBriefs in Applied Science and Technology, Springer-Heidelberg-Dordrecht-London-New York, 2011.

[10] Jaiani, G., Kharibegashvili, S., Natroshvili, D. and Wendland, W.L. Two-dimensional hierarchical models for prismatic shells with thickness vanishing at the boundary, J. Elasticity 77 (2), 95-122, 2004.

[11] Jaiani, G., Kufner, A. Oscillation of cusped Euler-Bernoulli beams and Kirchhoff-Love plates, Hacettepe Journal of Mathematics and Statistics, 35 (1), 7-53, 2006.

[12] Gordeziani, D.G. On the solvability of some boundary value problems for a variant of the theory of thin shells, Dokl. Akad. Nauk SSSR 215 (6), 1289-1292, 1974, in Russian.

[13] Opic, B. and Kufner, A. Hardy-type inequalities, Longman Sci. Tech., Harlow, 1990.

[14] Schwab, Ch. A-posteriori modeling error estimation for hierarchic plate models, Numer. Math. 74 (2), 221-259, 1996.

[15] Vekua, I. On one method of calculating of prismatic shells, Trudy Tbilis. Mat. Inst. 21, 191-259, 1955, in Russian.

[16] Vekua, I. Shell theory: general methods of construction, Pitman Advanced Publishing Program, Boston-London-Melbourne, 1985.

[17] Vishik, M. I. Boundary value problems for elliptic equations degenerating of the boundary of domain, Math. Sb. 35 (77, 3), 513-568, 1954, in Russian. 
